\title{
Colecistectomía laparoscópica por colecistitis en vesícula biliar siniestra incidental. Reporte de dos casos
}

\author{
Laparoscopic cholecystectomy in cholecystitis with incidental Left-sided \\ gallbladder. Two case report
}

Francisco Terrazas Espitia, * Pedro Arellano Martínez, ${ }^{* *}$ David Molina Dávila**

Palabras clave: Vesícula biliar, vesícula biliar ectópica, vesícula biliar izquierda, colecistectomía laparoscópica, laparoscopia.

Key words: Gallbladder, ectopic gallbladder, left-sided gallbladder, laparoscopic cholecystectomy, laparoscopy.

* Cirujano adscrito del Servicio de Cirugía Digestiva.

** Cirujano Asociado del

Servicio de Cirugía.

Hospital Español de

México, Ciudad de

México.

Recibido: 26/02/2016

Aceptado: 09/01/2017

\section{RESUMEN}

Objetivo: Presentar dos casos de vesícula biliar siniestra así como una revisión de la literatura disponible en la actualidad. Descripción de caso: Caso 1. Masculino de 64 años con cuadro de dolor abdominal tipo cólico en hipocondrio derecho de dos horas de evolución que remite con analgesia no especificada. Se realiza ultrasonido abdominal superior que evidencia poliposis vesicular, litiasis vesicular de pequeños elementos sin identificar e implantación anómala de la vesícula biliar. Se lleva a cabo cirugía laparoscópica y se identifica transoperatoriamente vesícula biliar de implantación izquierda. Caso 2. Paciente femenino de 33 años con antecedente de embarazo de 16 semanas de gestación. Inicia padecimiento actual ocho horas previo a su ingreso con cuadro de dolor en epigastrio e hipocondrio derecho irradiado a tórax posterior. Se realiza ultrasonido abdominal superior con evidencia de vesícula biliar con pared $4 \mathrm{~mm}$, múltiples litos así como lodo biliar con presencia de doble halo perivesicular y dilatación de vía biliar intrahepática leve. Se inicia manejo antibiótico y analgésico y tres días posterior a su ingreso se lleva a cabo colecistectomía laparoscópica, identificando implantación de vesícula biliar al lado izquierdo del ligamento redondo. La paciente presentó una evolución adecuada postoperatoria y fue dada de alta del servicio tres días después. Conclusión: La presencia de una vesicula biliar ectópica debajo del lóbulo izquierdo del hígado sin situs inversus es una variante anatómica poco común, conocida como vesícula biliar siniestra que se manifiesta entre 0.3 y $1 \%$ de la población que con frecuencia es un hallazgo transquirúrgico. Se presenta el reporte de dos casos de esta patología y una revisión de la bibliografía disponible.

\section{ABSTRACT}

Objective: To present two cases of left-sided gallbladder as well as a review of the literature available today. Case presentation: Case 1. 64 year old male with right upper quadrant pain with two hour duration, which diminishes after administrating unspecified NSAIDs. The upper abdominal ultrasound shows a gallbladder with a polyp and multiple small stones, without any radiologic evidence of anomalous gallbladder implanting. During laparoscopic cholecystectomy we identify a left-sided gallbladder. Case 2. 33-year-old female patient with history of being 16 weeks pregnant, is admitted with an eigth hour abdominal pain in the right upper quadrant which irradiates to the back. Abdominal ecography scan shows a gallbladder with multiple gallstones, a $4 \mathrm{~mm}$ wall, perivesicular liquid collections and dilation of intrahepatic ducts, but not a left-sided gallbladder implanting. Antibiotics and analgesics are started and three days later, she undergoes laparoscopic cholecystectomy. During the procedure, we identify a gallbladder implanted on the left side of the round ligament. The patient had an adequate evolution and was discharged three days later. Conclusion: The presence of an ectopic gallbladder located under the left lobe of the liver without situs inversus totalis is known as a left-sided gallbladder, and found in 0.3 to $1 \%$ of total population, usually transoperatively. We present two cases and a revision of the literature currently available.

\section{INTRODUCCIÓN}

$\mathrm{L}$ a vesícula biliar se origina por la cavitación y la coalescencia de las células del intestino primitivo, simultáneas a la formación del hígado. Existen diferentes alteraciones congénitas de la vesícula como son: 1) vesícula biliar septada, 2) vesícula biliar doble con conducto cístico separado o común, 3) conductos císticos accesorios, 4) vesícula biliar intrahepática y 5) 
vesícula biliar en situación siniestra. ${ }^{1}$ La vesícula biliar siniestra es una anomalía congénita inusual que se define como la implantación de la misma en la superficie inferior del segmento lateral izquierdo del hígado. Esta vesícula biliar se sitúa debajo del lóbulo hepático izquierdo, entre los segmentos III y IV o en el segmento III a la izquierda del ligamento falciforme. Es una condición parafisiológica que fue descrita por primera vez en 1886 por Horchstetter ${ }^{2}$ y está relacionada con 3 patologías: situs inversus, vesícula biliar ectópica bajo el lóbulo izquierdo y ligamento redondo en el lóbulo derecho. Con frecuencia se asocia a otras anomalías como las alteraciones en el sistema porta, las alteraciones del árbol biliar y la atrofia del segmento IV. ${ }^{3}$ Cuando se identifica previo a la cirugía, esta condición debe ser estudiada de forma exhaustiva utilizando tomografía computarizada o resonancia magnética. Cuando el hallazgo es transoperatorio, debe identificarse adecuadamente y predecir la confluencia aberrante del conducto cístico con la vía biliar común. Si surgen dudas en el diagnóstico, el cirujano deberá realizar una colangiografía transoperatoria que permita delimitar adecuadamente la anatomía de la vía biliar.

En el presente artículo se reportan dos adultos con hallazgo incidental transoperatorio de vesícula biliar siniestra durante colecistectomía laparoscópica con un manejo exitoso de la patología.

\section{REPORTE DE CASOS}

Caso 1. Paciente masculino de 64 años de edad, sin antecedentes personales patológicos de importancia quien inicia padecimiento actual ocho días previo a su ingreso con cuadro clínico de dolor abdominal tipo cólico en hipocondrio derecho de dos horas de duración que desaparece totalmente con analgesia no especificada. Niega antecedentes de cuadros similares previos. Se realiza ultrasonido que revela pólipo de $10 \mathrm{~mm}$ de diámetro y litiasis vesicular de pequeños elementos sin identificar y localización anómala de la vesícula biliar, por lo que se programa para colecistectomía laparoscópica de forma electiva. Se realiza cirugía laparoscópica con 3 trocares colocados en epigastrio, hipocondrio y flanco derecho y durante el transcurso de la cirugía se identifica vesícula biliar siniestra, localizada en el segmento III hepático, del lado izquierdo del ligamento suspensorio hepático (Figuras 1 a 3). Se decide no agregar trocares auxiliares, llevando a cabo colecistectomía con colocación de puertos de forma convencional identificando las diversas

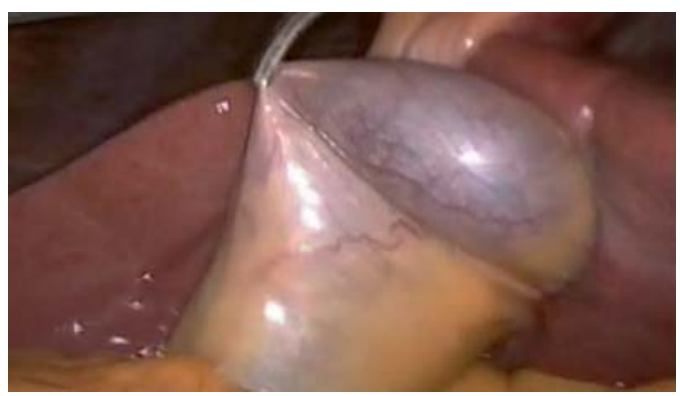

Figura 1. En esta foto se observa implantación de la vesícula biliar en el segmento III hepático y del lado izquierdo del ligamento suspensorio.

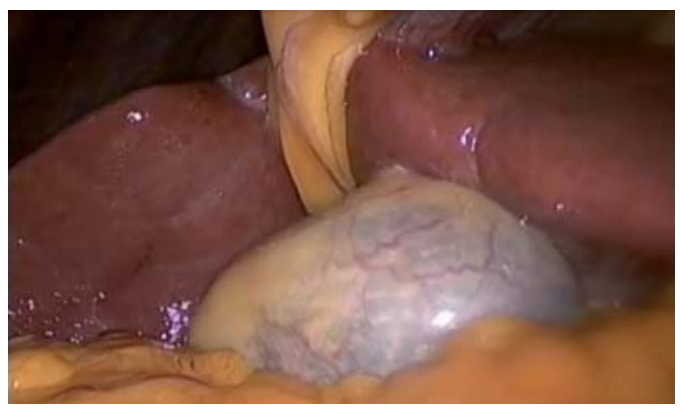

Figura 2. Foto que muestra la implantación anómala de la vesícula biliar al lado izquierdo del ligamento suspensorio.

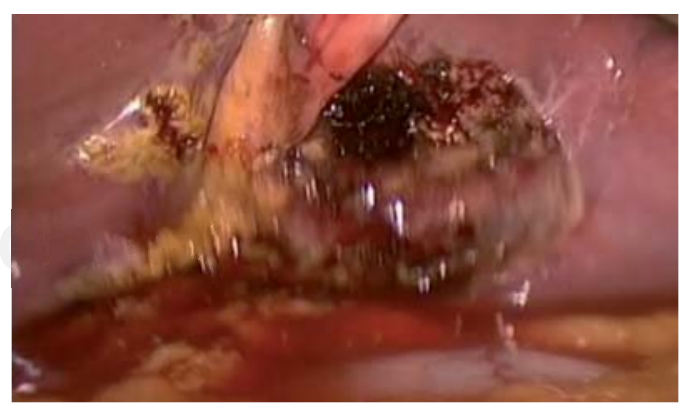

Figura 3. Foto que en la que se aprecia el lecho vesicular posterior a colecistectomía; confirmando la relación anatómica descrita con anterioridad. 
estructuras anatómicas sin accidentes ni incidentes, reportando un tiempo quirúrgico de una hora.

Caso 2. Paciente femenino de 33 años con embarazo de 16 semanas de gestación. Inicia padecimiento actual 8 horas previas con dolor tipo cólico 8/10 en epigastrio e hipocondrio derecho irradiado a espalda en hemicinturón derecho, acompañado de náusea y vómito. Se realiza ultrasonido abdominal superior que evidencia una vesícula biliar de $9 \times 5 \mathrm{~cm}$ de diámetro, con un grosor de pared de $4 \mathrm{~mm}$, múltiples litos y lodo biliar, así como presencia de doble halo perivesicular y dilatación de la vía biliar intrahepática. Se inicia manejo médico endovenoso mediante analgésico (antiiflamatorios no esteroideos) y antibiótico (cefalosporina de tercera generación); tres días después se lleva a cabo procedimiento quirúrgico. Se realiza laparoscopia con colocación de trocares de trabajo en epigastrio, hipocondrio y flanco derecho así como un trocar óptico (12 $\mathrm{mm}$ ) periumbilical. Se identifica vesícula biliar con implantación a la izquierda del ligamento redondo, específicamente sobre segmento III hepático (Figuras 4 y 5). No hubo necesidad de colocar puertos de trabajo agregados a los previamente colocados (de acuerdo a la técnica convencional). Se reportó un tiempo quirúrgico de 2 horas. Sin accidentes ni incidentes transoperatorios.

\section{DISCUSIÓN}

El origen embriológico de la vesícula biliar se encuentra en la región anterior de la porción caudal del intestino anterior en la cual durante el día 18 de gestación se forma un brote endodérmico. Éste se divide en 2 porciones: una cefálica de mayor tamaño que dará origen al parénquima hepático, a las vías biliares intrahepáticas y a los conductos hepáticos. La porción caudal que es más pequeña dará origen a la vesícula biliar y al conducto cístico. Por este origen la vesícula biliar se localiza por debajo de la unión del lóbulo cuadrado y el lóbulo derecho. La vesícula biliar izquierda se refiere a aquélla situada a la izquierda del ligamento redondo. Existen varias teorías sobre el origen de esta alteración, principalmente relacionadas con la forma en la que el conducto cístico desemboca en el conducto hepático común. La primera menciona que existe un desarrollo normal de la vesícula, pero durante su migración presenta una rotación anterior y hacia el lóbulo izquierdo, llevándose finalmente una implantación definitiva entre los segmentos III y IV. En ésta se observa al conducto cístico circundar el conducto hepático común hacia el lado izquierdo y desembocar en el lado derecho de éste. ${ }^{3,4}$ Otra teoría explica que durante el desarrollo embriológico se origina un brote endodérmico del lado izquierdo y degeneración del brote derecho; en este caso se observa el conducto cístico desembocar en el lado izquierdo del conducto hepático común. ${ }^{5}$ La vesícula biliar izquierda puede igualmente originarse del conducto hepático izquierdo, presentándose en estos casos asociado a alteraciones en la anatomía de la vía biliar y del sistema porta. ${ }^{3}$ En otros casos la vesícula biliar se encuentra en el lecho vesicular habitual,
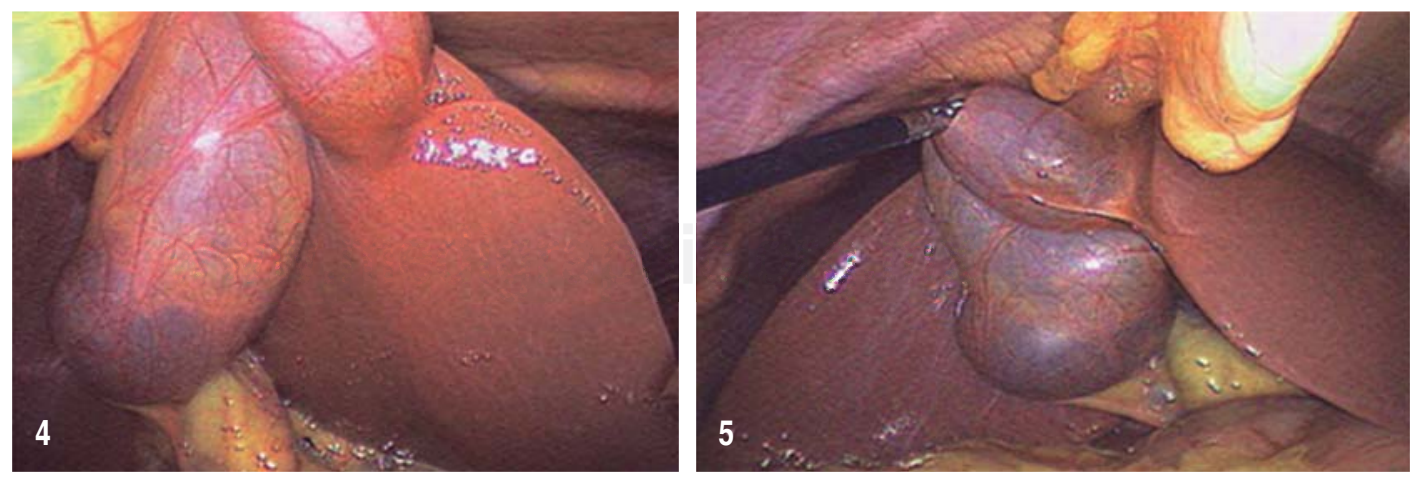

Figuras 4 y 5. Obsérvese posición de la vesícula implantada en segmento III hepático con lateralidad izquierda al ligamento suspensorio hepático. 
pero se presenta una desviación a la derecha del ligamento redondo. ${ }^{4}$ Esto ocurre porque durante el desarrollo embrionario existen los ligamentos umbilicales derecho e izquierdo. Normalmente el ligamento derecho se atrofia y el izquierdo se vuelve dominante. En raros casos esto sucede al revés. En estos casos la vesícula biliar se encuentra en su sitio habitual, pero el ligamento se sitúa más hacia el lóbulo derecho, por lo que ésta aparenta estar situada en el lóbulo izquierdo. ${ }^{6}$

Algunos autores han sugerido que las colecistectomías laparoscópicas en pacientes con vesícula siniestra se realicen colocando los puertos operatorios de manera inversa a la técnica convencional (epigastrio/hipocondrio izquierdo/flanco derecho) o colocando un puerto extra en el hipocondrio izquierdo. ${ }^{7,8} \mathrm{Sin}$ embargo, la mayoría de las vesículas biliares izquierdas no son posibles de diagnosticar previo a la cirugía con ultrasonido ni con CPRE, razón por la que es difícil tomar estas medidas. ${ }^{6} \mathrm{El}$ hallazgo transoperatorio de esta alteración debe alertar al cirujano sobre posibles alteraciones de la anatomía de la vía biliar o localización de la arteria cística, ${ }^{3,4}$ en consecuencia deberá tenerse mayor precaución durante la disección del conducto y arteria cística para evitar lesiones en estas estructuras. Zografos et al. refieren que desde el punto de vista estadístico la necesidad de realizar conversión a cirugía abierta es exponencialmente mayor en presencia de vesícula siniestra en comparación con aquellos procedimientos en los que no se observa dicha alteración. ${ }^{7}$ El único reporte encontrado en la literatura hispana fue realizado por Morales Ricardo et al. en Almería, España. En esta referencia el equipo médico requirió llevar a cabo conversión a cirugía abierta debido a la dificultad de localizar la vesícula biliar e identificar adecuadamente las estructuras anatómicas críticas para la conclusión del procedimiento. ${ }^{9}$

\section{CONCLUSIÓN}

La vesícula biliar siniestra es una entidad poco frecuente y por lo general es un hallazgo trans- operatorio. El diagnóstico preoperatorio o transoperatorio de la patología deberá alertar al cirujano sobre variantes anatómicas probables de la vía biliar y del sistema porta para, de esta manera, tomar las precauciones necesarias con el fin de evitar lesiones incidentales o, en su caso, realizar conversión a cirugía abierta para garantizar la seguridad del paciente.

\section{REFERENCIAS}

1. Dhulkotia A, Kumar S, Kabra V, Shukla HS. Aberrant gallbladder situated beneath the left lobe of liver. HPB (Oxford). 2002; 4: 39-42.

2. Hochstetter F. Anomaliem der Pfortader und der Nabelvene in verbindung mit Defect oder Linkscage der Gall-en-blasé. Archiv für mikroskopische Anatomie und Entwicklungsgeschichte, 1886, pp. 369-384.

3. Hsu SL, Chen TY, Huang TL, Sun CK, Concejero AM, Tsang LL, Cheng YF. Left-sided gall bladder: Its clinical significance and imaging presentations. Worold J Gastroenterol. 2007; 13: 6404-6409.

4. Nagai M, Kubota K, Kawasaki S, Takayama T, Bandai Y, Makuuchi M. Are left-sided gallbladders really located on the left side? Ann Surg. 1997; 225: 274-280.

5. Newcombe JF, Henley FA. Left-Sided Gallbladder. A review of the literature and a report of a case associated with hepatic duct carcinoma. Arch Surg. 1964; 88: 494-497.

6. Qureshi I, Awad Z. Aberrant presentation of the gallbladder during laparoscopic cholecystectomy. JSLS. 2009; 13: 605-607.

7. Zografos G, Lagoudianakis E, Grosomanidis D, Koronakis N, Tsekouras D, Chrysikos J, et al. Management of incidental left-sided gallbladder. JSLS. 2009; 13: 273-275.

8. Matsumura N, Tokumura H, Yasumoto A, Sasaki $\mathrm{H}$, Yamasaki M, Musuya H, et al. Laparoscopic cholecystectomy and common bile duct exploration for cholecystocholedocholithiasis with a left-sided gallbladder: report of a case. Surg Today, 2009; 39: 252-255.

9. Zango BA, Morales SP, Raimundo MG, Pinillos JL, Paulino AM, Nácher JB. Correct placement of left-sided double lumen endotracheal tubes: a simple verification technique. Rev Esp Anestesiol Reanim. 2008; 55: 277 281.

\section{Correspondencia:}

Dr. Francisco Terrazas Espitia

Av. Ejército Nacional Núm. 613,

Col. Granada, Del. Miguel Hidalgo,

11520, Ciudad de México, México.

Tel. (55) 52-55-96-00

E-mail: franciscoter6@hotmail.com 